\title{
A Feasibility Study of Hand Kinematics for EVA Analysis Using Magnetic Resonance Imaging
}

Reuben D. Dickenson Vanderbilt Univ.

Christine H. Lorenz

Vanderbilt Medical Center

Steven W. Peterson, Alvin M. Strauss, and John A. Main Vanderbilt Univ.

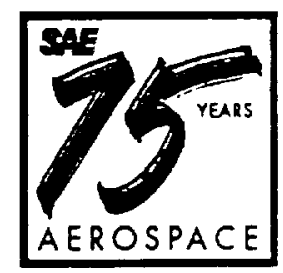
The Engineering Society INT Land Sea Air and Space

22nd International Conference on Environmental Systems Seattle, Washington July 13-16, 1992

400 COMMONWEALTH DRIVE, WARRENDALE, PA $15096-0001$ U.S.A. 
The appearance of the ISSN code at the bottom of this page indicates SAE's consent that copies of the paper may be made for personal or internal use of specific clients. This consent is given on the condition, however, that the copier pay a $\$ 5.00$ per article copy fee through the Copyright Clearance Center, Inc. Operations Center, 27 Congress St.. Salem, MA 01970 lor copying beyond that pemitted by Sections 107 or 108 of the U.S. Copyright Law. This consent does not extend to other kinds of copying such as copying for general distribution, for advertising or promotional purposes, for creating new collective works, or for resale.

SAE routinely stocks printed papers for a period of three years following date of publication. Direct your orders to SAE Customer Service Department.

To obtain quantity reprint rates, permission to reprint a technical paper or permission to use copyrighted SAE publications in other works, contact the SAE Publications Group.

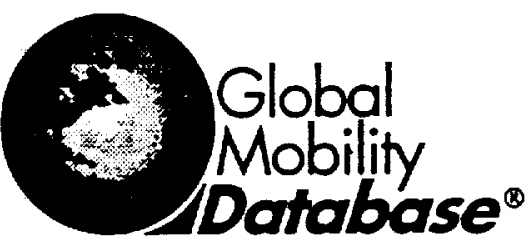

All SAE papers, standards, and selected books are abstracted and indexed in the SAE Global Mobility Database.

No part of this publication may by reproduced in any form, in an electronic retrieval system or otherwise, without the prior written permission of the publisher.

ISSN 0148-7191

Copyright 1992 Society of Automotive Engineers, Inc.

Positions and opinions advanced in this paper are those of the author(s) and not necessarily those of SAE. The author is solely responsible for the content of the paper. A process is available by which discussions will be printed with the paper if it is published in SAE transactions. For permission to publish this paper in full or in part, contact the SAE Publications Division.

Persons wishing to submil papers 10 be considered for presentation or publication through SAE should send the manuscript or a 300 word abstract of a proposed manuscript to: Secretary, Engineering Activity Board, SAE.

\section{Printed in USA}




\section{A Feasibility Study of Hand Kinematics for EVA Analysis Using Magnetic Resonance Imaging}

Reuben D. Dickenson

Vandertilt Univ.

Christine H. Lorenz

Vanderbilt Medical Center

Steven W. Peterson, Alvin M. Strauss, and John A. Maln

Vanderbilt Univ.

\section{ABSTRACT}

A new method of analyzing the kinematics of joint motion is developed in this study. Magnetic Resonance Imaging (MRI) offers several distinct advantages. Past methods of studying anatomic joint motion have usually centered on four approaches. These methods are $x$-ray projection, goniometric linkage analysis, sonic digitization, and landmark measurement of photogrammetry. of these four, only $x$-ray is applicable for in vivo studies. The remaining three methods utilize other types of projections of inter-joint measurements, which can cause various types of error. MRI offers accuracy in measurement due to its tomographic nature (as opposed to projection) without the problems associated with $\mathrm{x}$-ray dosage.

Once the data acquisition of MR images was complete, the images for this study were processed using a 3$D$ volume rendering workstation. In this study, the metacarpalphalangeal (MCP) joint of the left index finger was selected and reconstructed into a three-dimensional graphic display. From the reconstructed volumetric images, measurements of the angles of movement of the applicable bones were obtained and processed by analyzing the screw motion of the MCP joint. Landmark positions were chosen at distinctive locations of the joint at fixed image threshold intensity levels to ensure repeatability. The primarily twodimensional planar motion of this joint was then studied using a method of constructing coordinate systeins using three (or more) points. A transformation matrix based on a world coordinate system described the location and orientation of a local target coordinate system.

This study will lead to future research involving volume rendering of MRI data focusing on the internal kinematics of the hand's individual ligaments, cartilage, tendons, etc. Its findings will show the applicability of MRI to joint kinematics for gaining further knowledge of the hand-glove (powerassisted) design for EVA.

\section{INTRODUCTION}

The purpose of this research is to determine the feasibility of using Magnetic Resonance Imaging (MRI) as a tool for the study of hand kinematics. This project was funded by the NASA Office of Aeronautics and Space Exploration and the NASA National space Grant College and Fellowship Program. Various methods of determining the internal motion of the hand's internal bone structures exist, but MRI offers several advantages which will be discussed in detail later in this article. 
DATA ACQUISITION METHODS

The data for this study came from the images of the left hand of a 29 year old male. The entire hand was imaged, but for the purposes of this study, only the index finger was analyzed. The study focused on the rotation and translation characteristics of the proximal phalanx and its relation to the MCP joint, so that only the portions of the images pertaining to these areas were used. A restraint device was constructed to ensure that both random hand motion and vibratory noise were minimized during imaging. Although random hand motion can vary from one individual to the next, the magnitude of this motion is very small. Over the image acquisition period, this type of motion results in negligible position error. The device was designed to ensure that the index finger was separated from the other fingers in a fixed position. Six preset angles of rotation were possible using this device, of which three were used for this study. Since MR images' signal intensity is based on density of certain atoms (hydrogen in this case), markers containing water were placed on the restraint. Two of the markers were attached to the bottom of the device, while the third was attached to the hinged portion, just past the tip of the index finger (Fig 2) The markers appear as circles in Figure 2, with $A$ denoting the marker beneath the hinge, B showing the marker affixed to the tip of the base, and $C$ showing the marker at the fingertip. These markers allowed calibration of the procedure and a means for determining the accuracy of the group's results.

MRI is used widely for its tomographic capabilities, high spatial resolution, and ability to produce high contrast between different tissue types. The physics of MRI are among the most complicated of all the current imaging modalities $[2,3,7]$. Briefly, the basic MR process is to place a subject into a strong uniform magnetic field in the bore of a large magnet, which typically has a field strength on the order of 1.0-1.5 Tesla. This field aligns the net magnetic spin of the individual atoms. In order to produce a usable signal, these aligned magnetic spins must be perturbed. A radiofrequency ( $R F$ ) pulse is applied at the resonant frequency of the atomic spin of interest (hydrogen is usually chosen due to its abundance). The spins are moved away from their preferred axis of rotation by the energy imparted by the RF pulse. The spins will try to realign with their original axis of rotation (along the bore of the magnet), and as they realign, they emit a RF signal. This signal can be decomposed into its frequency and phase components which are related to the location in the subject from which the signal was measured. The magnitude of the signal is proportional to the signal intensity generated by the various structures in the image. This magnitude is, in turn, related to the density of hydrogen atoms in the tissue and the realignment (relaxation) parameters of the tissue studied. These parameters are called $T 1$, the longitudinal relaxation time, and $\mathrm{T} 2$, the transverse relaxation time. The density of the hydrogen atoms determines the material present in a give 3-D volume of space. This information is then projected onto a 2-D display for viewing (Figure 1). The image data can be manipulated to reorient the slices in different orientations for further analysis.

The programs used to control the MR scanner to create images are called pulse sequences. Each type of pulse sequence provides different contrast between various tissue types or has different spatial resolution or signal-to-noise properties. The most traditional (and slowest) type of pulse sequence is the spin echo pulse sequence. There are also many pulse sequences designed to provide essentially the same tissue contrast or signal-tonoise ratio as spin echo sequences, 


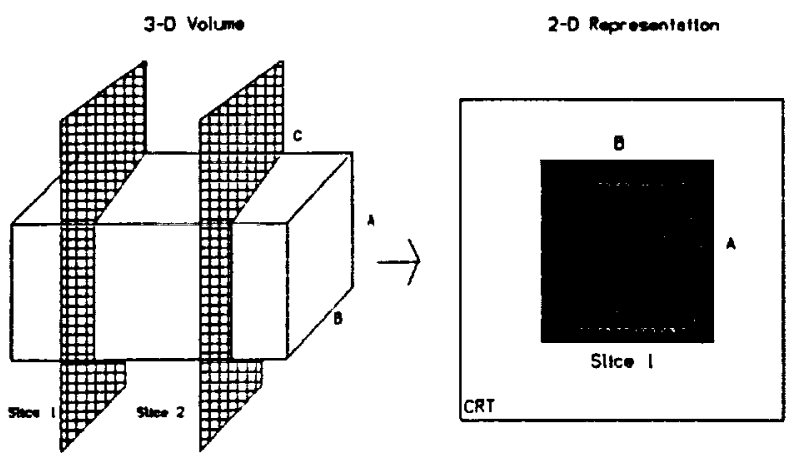

Figure 1 - Diagram showing the 3-D slices of a volume depicted on a 2-D CRT display.

but in a fraction of the time. Since our goal was to optimize speed, image quality, resolution, and tissue contrast, we used a variation of a fast pulse sequence called TurboFLASH. The scanner used was a $1.5 \mathrm{~T}$ clinical unit (Siemens Magnetom SP, Iselin, NJ). In order to obtain approximately isotropic image volume elements (voxels), we used a volumetric acquisition version of TurboFLASH known as MPRAGE (Magnetization Prepared Rapid Acquisition Gradient Echo). With this sequence, we obtained acceptable spatial resolution

(1-2 mm) images through the entire hand with good differentiation between tissue types, and an acceptable signal-to-noise ratio in approximately 7 minutes. The pulse sequence parameters used were TR $=6$ msec, flip angle $=10$ degrees, acquisition matrix $=130 \times 256 \mathrm{x}$ 128, and a field of view $=250 \mathrm{~mm}$.

Figure 1 demonstrates the tomographic capability of MRI. Since a volume of image data of up to $30 \mathrm{~cm}^{3}$ can be acquired using the particular radiofrequency coil chosen for this study, imaging the hand in an EVA glove will be a simple extension to this study.

\section{3-D VOLUME PREPARATION}

After acquiring the MRI data, it was necessary to prepare the volumes. These were created by adding the area of interest (the phalangeal bones of the index finger) for each slice of MRI data. Since each slice contains a 2-D representation of the phalanges, adding them creates the composite 3-D volume. All volume rendering was completed utilizing a DMI MAXIVIEW 3-D dedicated workstation (MAXIVIEW, Dimensional Medicine, Inc., Minnetonka, MN) and its accompanying software.

The MAXIVIEW workstation was created specifically as a tool for processing medical imaging modality data. It accepts image data from Computed Tomographic (CT) systems, Digital Subtraction Angiography (DSA) systems, and MRI systems. This system allows $3-D$ reformatting, resectioning and multi-planar reformatting for processing images. In addition to creating the volumes, MAXIVIEW also proved valuable for viewing, calibrating, and measuring the volumes.

The overall problem with the thresholding of MRI data during volume rendering deserves further discussion. The reconstruction sequence of computer workstations such as the MAXIVIEW depends largely upon its ability to extract as much surface detail as possible in two dimensions (ie, differentiate between the surface of the proximal phalanx and the surrounding tissues in a given slice) and to then add these surfaces for a 3-D representation (Vannier and Geist, [10]) using MRI, CT, or DSA data. However, as the resolution of the individual slices decreases, the resolution of the corresponding volume likewise decreases. This fact makes CT an excellent choice for rendering. It is based on attenuation values for $X$-rays, called Hounsfield numbers, which offer a stark contrast between bone and soft tissue. Bowever, CT still depends on X-ray attenuation, which makes MRI attractive for repetitive 
studies of this type, owing to its lack of ionizing radiation. But MRI suffers from its lower resolution of the border between bone and soft tissue (compared to CT), which often leads to difficulties in thresholding. This will become less of a problem as the contrast of MRI scanners' images increases.

Thresholding the images is the key step in rendering volumes. The images contain gray scale computed information based on a combination of hydrogen density, $\mathrm{T} 1$ and $\mathrm{T} 2$ relaxation times (MRI) or Hounsfield numbers (CT). This gray scale information is then utilized to determine what material is present inside a 3-D voxel. Voxels which contain the correct values are retained for inclusion in the volume, while others remain blank. It follows that the size of the voxel itself proves to be an overall limit to the volume's resolution. In this study, the typical volume was approximately 0.4 $\mathrm{mm}$ in each dimension $(x, y, z)$.

After the volume is computed, the next step is the shading process. An initial viewing direction is chosen. The 3-D view in this direction' is projected onto a 2-D plane, called the image plane, for display on the CRT.

Rendering the phalanges for this study was the next step. In an effort to maximize the resolution of the volumes, the markers and the bones were rendered separately. This created a separate representation for each, but the positions relative to each other remained fixed in the global coordinate system of the MRI scanner. For each slice of a pose, the appropriate bones were selected and thresholded. A second set of bone markers was added to the bone's rendered volume to ensure that the volume was correctly aligned while measuring in the $3-D$ mode (see Figures 3-5). After the volumes for the bones were created, the volumes for the restraint device's water markers were computed. The water markers served to calibrate and validate the measurements and algorithms for this study. A second set of markers were also produced with the water marker volumes to ensure alignment, just as we did with the phalanx volumes.

In order to analyze the motion of rigid bodies, we used the calculations and methods devised by several authors $[4,8,9]$. The spatial coordinates of landmark positions (a minimum of three on each rigid body in each position) can be calculated from the measurement tools of the DMI. These coordinates then comprise the information necessary to derive the transformation matrix describing the motion, which is taken to be a screw motion. A screw motion can be described by a rotation matrix and a translation vector, or by the position of the screw axis, the angle of rotation about this axis, and the translation along this axis. This method is a fairly robust method of determining the motion of the rigid body, but does suffer from errors induced by scaling errors and random measurement errors (noise). While a comprehensive treatment of the derivation of Spoor and Veldpaus' methods is not feasible in this study, several key points need to be clarified. First, for this method, three or more non-collinear points are needed in the first and second positions, with no initial approximation needed. The translation vector $(v)$ and the rotation matrix (R) describe the movement of a rigid body from position 1 to position 2 . The rotation matrix has the following properties:

$$
R^{T} R=I
$$

where $I$ is the $3 \times 3$ identity matrix and $T$ denotes transposition. The vectors describing each point in the first position $\left(a_{i}\right)$ and those describing the points in the second position $\left(q_{i}\right)$ are related by

$$
q_{i}=R a_{i}+v
$$

Since the unknown quantities in (2) are $R$ and $v$, a method must be used 


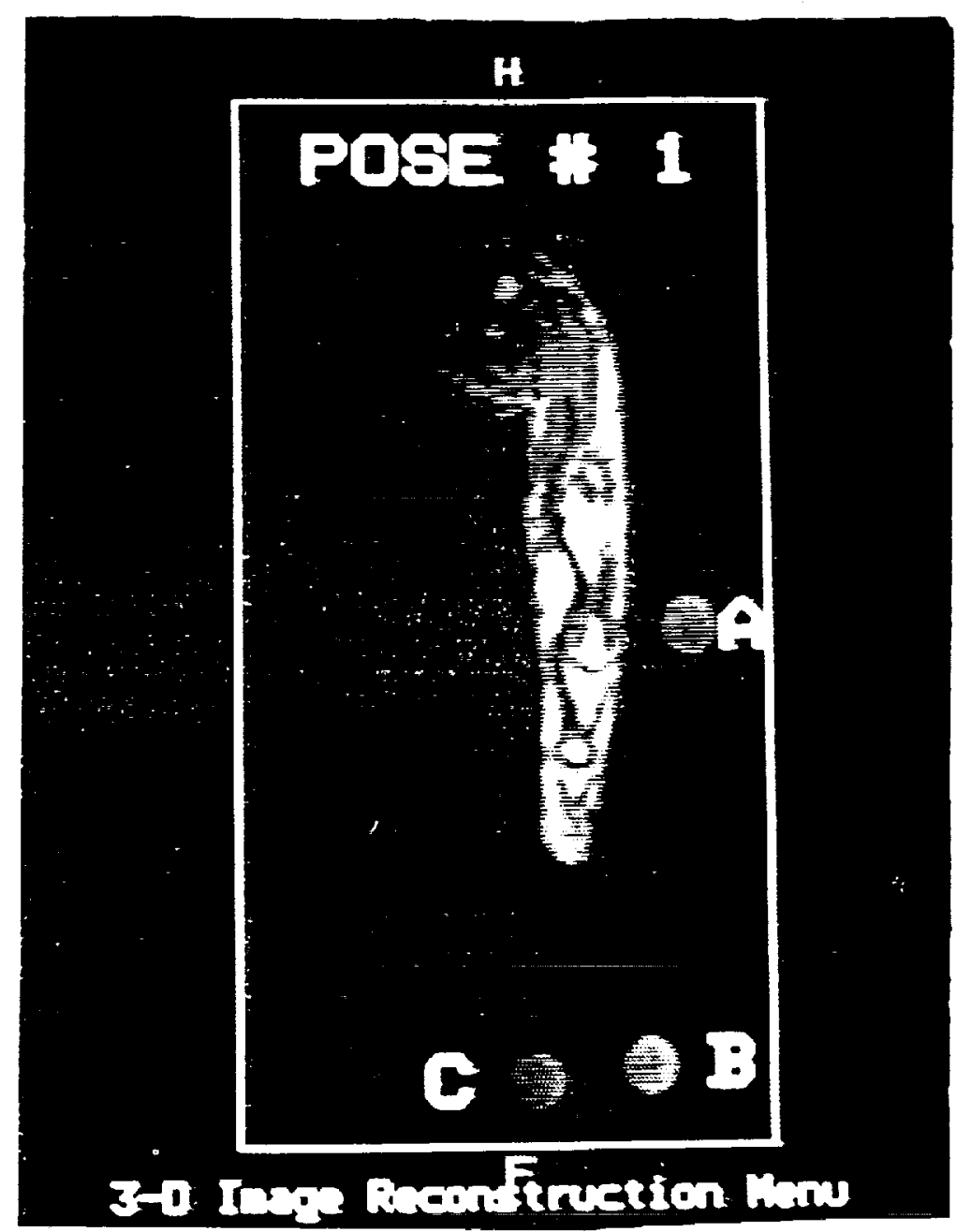

Fig 2 - A representative image of a "slice" of the left index finger in the restraint device. $A, B$, and $C$ show the markers.

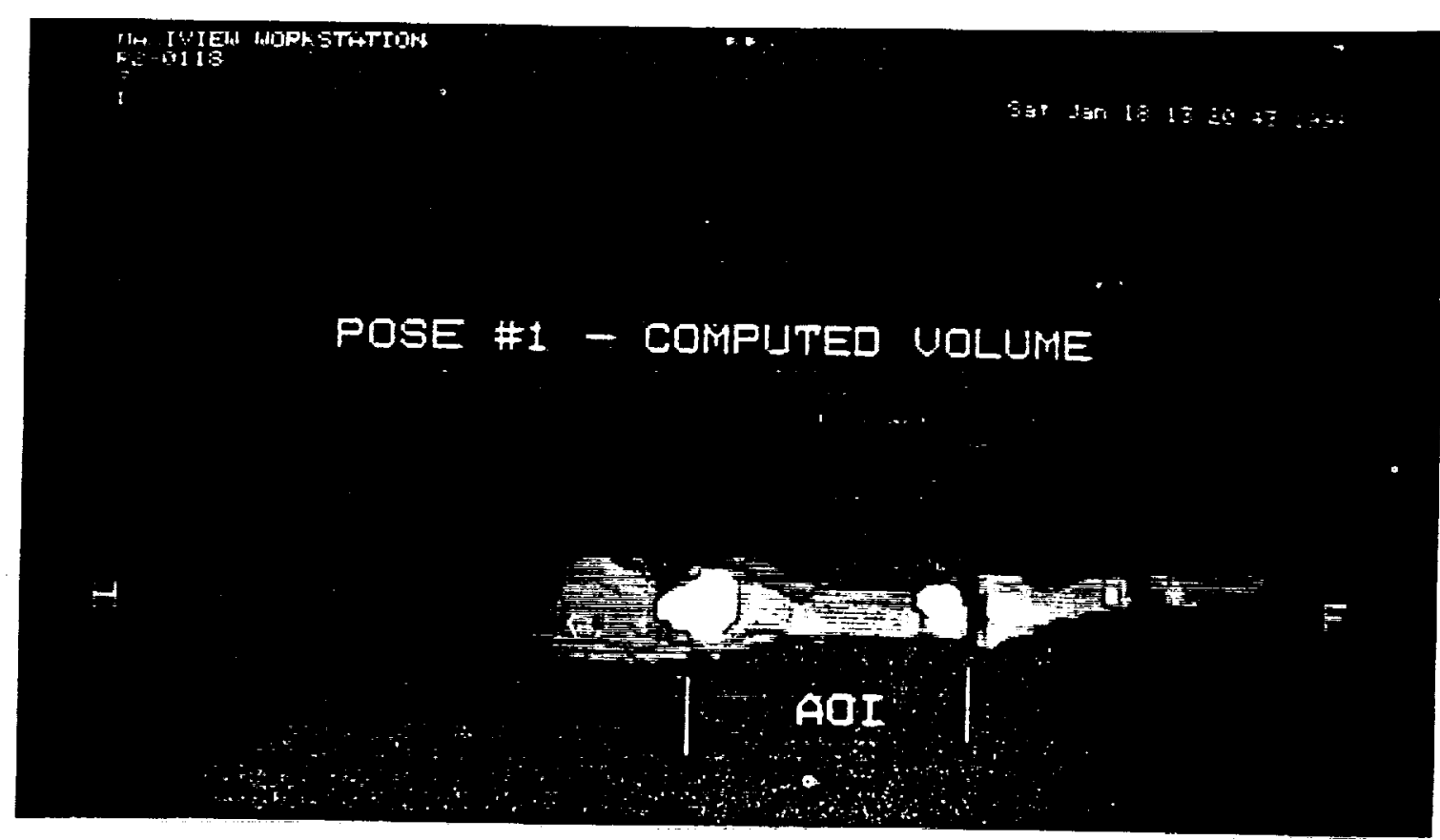

Fig 3 - The rendered volume in the first pose. 


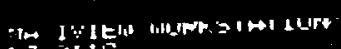

$=\frac{i}{2}+11: 3$

:

POSE \#2 - COMPUTED UOLUME

$H$

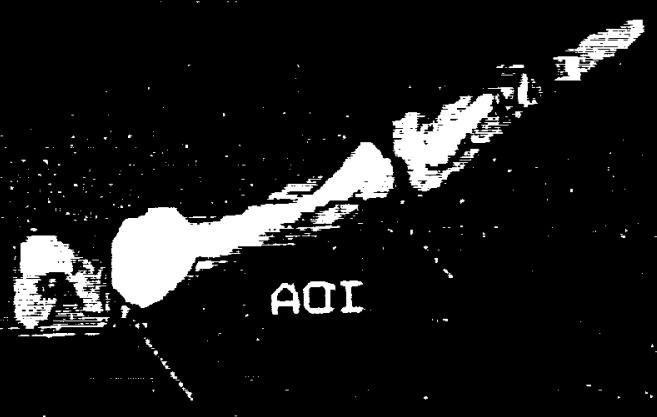

Fig 4 - The rendered volume in the second pose.

POSE \#3 - COMPUTED UOLUME

4

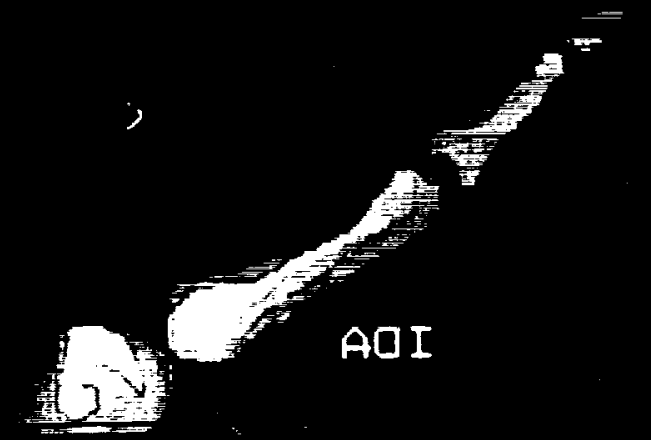

Fig 5 - The rendered volume in the third pose. 
to obtain values for them by using the measurements of the points in the first and second positions. A computer program was written (Peterson, [8]) which takes the three-dimensional coordinates from two positions of the rigid body and then computes the transformation matrix in the form of

$$
T=\left|\begin{array}{llll}
R_{11} & R_{12} & R_{13} & 0 \\
R_{21} & R_{22} & R_{23} & 0 \\
R_{31} & R_{32} & R_{33} & 0 \\
v_{1} & v_{1} & v_{1} & 1
\end{array}\right|
$$

where the $3 \times 3$ matrix in the upper left describes the rotation (R) of the body, and $v$ denotes the translation vector. Once the transformation matrix is determined, the methods of analyzing the motion can be one of several in existence. The method of Spoor and Veldpaus utilizes the symmetrical and antisymmetrical portions of the rotation matrix to determine the body's motion about the helical axis, with

$$
2 * n * \sin (\theta)=\left|\begin{array}{l}
R_{32}-R_{23} \\
R_{21}-R_{32} \\
R_{21}-R_{22}
\end{array}\right|
$$

The angular rotation is given by

$$
\sin (\theta)=1 / 2 \sqrt{\left(R_{32}-R_{23}\right)^{2}+\left(R_{13}+R_{31}\right)^{2}+\left(R_{21}-R_{12}\right)^{2}}
$$

The rotation is also given by

$$
\cos (\theta)=(1 / 2) *\left(R_{11}+R_{22}+R_{33}-1\right)
$$

Now that the angle is known, returning to equation (4) yields (n), the unit vector along the helical axis.

For the translation analysis, one must again derive an expression based upon the transformation matrix. The translation vector, $v$, is taken from the transformation matrix. The following information determines the necessary information:

$$
\begin{aligned}
{[1-\cos (\theta)] n n^{T}=[(R+R T)-\cos (\theta) T] / 2 } \\
\\
=\left|b_{1} b_{2} b_{3}\right|
\end{aligned}
$$

where $b_{i}$ are three element columns. Another value for $\mathbf{n}$ is determined from

$$
b_{1}^{T}=\max \left(b_{2}^{\top} b_{1}, b_{2}^{T} b_{2}, b_{3}^{\top} b_{1}\right) ; n= \pm \frac{b_{1}}{\sqrt{b^{T} b_{1}}}
$$

where $\mathbf{n}$ will be in the same direction as that derived from (4), but a scaling factor different in magnitude. The values for a point on the axis and the translation on the axis are then found from

$$
t=n^{x} * v
$$

and

$$
s=(-1 / 2) * n *(n * v)+\frac{\sin (\theta)}{(2(1-\cos (\theta))} * n * v
$$

where $\mathbf{s}$ represents a radius vector of a point on the helical axis, and $t$ is the translation along the helical axis. Noteworthy is the fact that the translation vector is quite sensitive to measurement errors and noise, while the rotation matrix is rather robust and yielded highly accurate results.

\section{DATA ANALYSIS/RESULTS}

The angles of rotation about the helical axis for the respective position changes for this study were

$$
\begin{aligned}
& \text { ANGLES OF ROTATION - POSE \#3 } \\
& \text { MARKERS } \\
& \text { Restraint Device - } 52.0 \mathrm{deg} \\
& \text { 2-D Slice Image }-52.9 \mathrm{deg} \\
& \text { 3-D Volume } \quad-52.7 \mathrm{deg}
\end{aligned}
$$

Table 1 - Calibration Results

quite accurate, as were the calibration results utilizing the markers. Each marker set for the poses was rendered into a 3-D volume, just as the phalanx voxels were rendered. However, the water marker volumes were created for one slice of image data only. This was 
to ensure that a clear rotation about one axis alone was visible, thus further validating the measurements of the DMI and the accuracy of the transformation matrix software. From the slice containing the markers (see Figure 2), measurements of the markers for pose \#3 were determined for the angle $B-A-C$. This angle was then compared to the angle produced by measuring landmark positions from the rigid body $A-B$ (notionally position 1) moving to A-C (notionally to position 2). The results are listed in Table 1. Furthermore, the unit vector along the helical axis for the rotation of Pose \#3 was $n=\left[\begin{array}{lll}0 & 0 & 1\end{array}\right]^{\mathrm{T}}$, thus showing that for the markers, a simple 2-D rotation occurred in the z-axis, which proves valuable later.

After checking the process using the water markers, we then measured and analyzed the rigid body motion of the landmark positions on the proximal phalanx. The measurements were taken in the $(x, y, z)$ coordinates and then transformed into the transformation matrix. From this, as in the case of the markers, we determined the motion of the phalanx about the helical axis using spoor and Veldpaus algorithms. Seven landmark positions defined each rigid body motion, with future efforts geared toward increasing the number of landmark positions for increased accuracy and reliability. The results are listed in Table 2 .

$$
\begin{aligned}
& \begin{array}{llll}
\text { From-To } & 1 & \underline{3}
\end{array} \\
& \text { (deg) }
\end{aligned}
$$

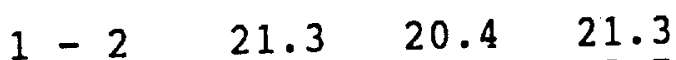

$$
\begin{aligned}
& \begin{array}{llll}
1-3 & 12.4 & 14.0 & 12.7
\end{array} \\
& \begin{array}{llll}
1-3 & 32.6 & 32.9 & 33.5
\end{array} \\
& \text { Table } 2 \text { - The 3-D rotations } \\
& \text { for three trials. }
\end{aligned}
$$

\footnotetext{
The overall consistency of the results above can be readily
}

observed. The fact that the accuracy of the transformation increases as the magnitude of motion increases is a point of interest for future work. Also, as in the case of the markers, the motion was primarily twodimensional about the z-axis, as expected. The small rotation in the $x$ and $y$ axes can be attributed mainly to the alignment of the subject hand. Random measurement errors also played a role. The mean and standard deviation of the movements are listed in Table 3 .

From-To Mean Deviation(deg)

$$
\begin{array}{lll}
1-2 & 21.1 & .8 \\
2-3 & 13.0 & .7 \\
1-3 & 33.5 & .4
\end{array}
$$

Table 3 - Statistical Analysis.

\section{CONCLUSION}

As noted earlier, future work in this area includes ongoing powerassisted glove work. A subject could wear the EVA glove inside the magnet, placing the hand within the radiofrequency coil. By stopping a subject at various stages of the motion required to perform a task, high resolution imaging of the entire had and glove volume could be performed at each stage. The transformations of the entire hand's members could then be analyzed. The effect of using the glove compared to non-glove motion could also be studied. Other areas of possible interest include those involving potential applications of injuryrelated analysis of the hand. One major subject in this field is study of individual joint kinematics leading to injury suffered during certain repetitive activities, such as those encountered by a computer programmer while entering data at a keyboard.

Our research proved that MRI is 
a feasible tool for kinematic analysis of rigid bodies. The process was very slow for this initial study, due to several factors, but subsequent studies will be completed much more quickly. The screw motion analysis of the proximal phalanx in this research produced results that were both reliable and repeatable for the proximal phalanx and should yield the same results for whole hand analysis, a future goal of the group. While other methods do exist, such as electrical goniometry, computed tomography, etc., only MRI offers a safe means of producing accurate results for in vivo analysis.

\section{REFERENCES}

1. Chao E.Y. and Morrey B.F., "Three-Dimensional Rotation of the Elbow", Journal of Biomechanics, Volume 11, pp. 57-73, 1978.

2. Haase, A. "Snapshot FLASH MRI. Applications to $\mathrm{T} 1, \mathrm{~T} 2$, and Chemical-Shift Imaging." Magnetic Resonance Medicine 1990;

$13: 77-89$.

3. Jones, J.P. "Physics of the MR Image: From the Basic Principles to Image Intensity and contrast, in Magnetic Resonance Imaging: Vol. II, Physical Principles and Instrumentation, eds. Partain, C.I. et al., W.B. Saunders Company, Philadelphia, PA PP. 1003-1028.

4. Kinzel G.L. et al., "Measurement of the Total Motion Between Two Body segments - I. AnalyticaI Development", Journal of Biomechanics, Volume 5, pp. 93-105, 1972 .

5. Kinzel G.L. et al., "Measurement of the Total Motion Between Two Body Segments - II. Description of Application", Journal of Biomechanics, volume 5, pp. 283-293, 1972 .

6. Larsen R.J. and Marx M.L., Mathematical statistics and Its
Applications, Prentice-Hall, Englewood Cliffs, New Jersey, 1986.

7. Mugler, J.P. and Brookeman J.R., "Three-Dimensional Magnetization Prepared Gradient Echo Imaging", society of Magnetic Resonance in Medicine Abstract Book, 1990 , p. 432 .

8. Peterson S.W., Measurement and Analysis of Human Joint Motion, Ph.D. Thesis, University of Minnesota, December 1985.

9. Spoor C.W. and Veldpaus F.E., "Rigid Body Motion Calculated from Spatial Co-ordinates of Markers", Journal of Biomechanics, Volume 13, pp. 391-393.

10. Vannier M.W. and Geist D., "PCBased 3-D Reconstruction of Medical Images", Computer and Graphics, Volume 13, No. 2, 1989, pp 135-143. 